\title{
Characterizing Single Complete Denture Opposing Natural Teeth and Partial Denture - Case Report
}

\author{
Khurshid $\mathrm{AM}^{1 *}$ and Sarvar $\mathrm{S}^{2}$ \\ ${ }^{1}$ Department of Prosthodontics, College of dentistry, Jazan University, Saudi Arabia \\ ${ }^{2}$ Senior Dental Technician, SVS University, India \\ *Corresponding author: Khurshid A Mattoo, Assistant Professor, Department of \\ Prosthodontics, College of dentistry, Jazan University, Saudi Arabia, E-mail: \\ drkamattoo@rediffmail.com
} \begin{tabular}{|c|}
\hline Case Report \\
Volume 2 Issue 4 \\
Received Date: September 25,2017 \\
Published Date: October 09,2017 \\
DoI: $10.23880 /$ oajds-16000147 \\
\hline
\end{tabular}

\begin{abstract}
Every human being is unique biologically. This not only implies to his biological make up but also to his behavior. When it comes to replacement of teeth, most patients that dentist encounters are over demanding in terms of esthetics of their new teeth. Expectations of patients that are realistic have to be met while ignoring unrealistic ones. A male patient reported with few natural teeth in the maxillary arch while being completely edentulous in the mandibular arch. Remaining natural teeth had undergone gingival recession and staining due to tobacco and tea. A single complete denture for mandibular arch and a treatment, partial denture for the maxillary arch was fabricated in which the denture teeth were characterized with staining.
\end{abstract}

Keywords: Poly methyl methacrylate; Complete denture; Denture stains; Gingiva

\section{Introduction}

Among various realistic expectations that completely or partially edentulous patients have, the will to have their denture look like their existing natural teeth is genuine. To accomplish such objectives, the art of characterization, especially staining of the artificial denture has evolved since 1951, when Pound introduced the technique depending on the racial and individual pattern [1,2]. Denture characterization implies a denture base with color that simulates the color and shading of natural oral tissues [3]. There are many methods that have been reported in the literature [4-7]. Although denture characterization has been mainly reported for complete dentures where denture characterization is subjective and cannot be compared, this report is unique that the characterization of single complete denture and partial denture had to match with the staining of existing natural teeth which were few in number.

\section{Case Report}

An elderly male patient, aged 72 years, reported to the department of Prosthodontics for early replacement of remaining natural teeth primarily for an esthetic purpose. Medical, social, dental and drug history were non contributory. Extra oral and intra oral examination did not reveal any negative findings that would modify the treatment plan. Maxillary arch had two central incisors, one lateral incisor and one premolar on the left side that were firm and had clinically normal surrounding structures (Figure 1a). Gingival recession and staining was present in relation to the central incisors with staining (light brown to dark brown) concentrated in the cervical region. After thorough investigations a diagnosis was made and various treatment plans were presented to 
the patient. Although Overdenture for maxillary arch was the first priority, the patient due to superstitious beliefs did not want to extract the front teeth. The treatment option that patient gave consent included maxillary interim partial denture and mandibular single complete denture. Both dentures were fabricated conventionally. However, during denture processing denture acrylic was stained using Replident (kit of 8 dispenser bottles of different colored methacrylate polymers). Accurate staining was achieved by first preparing an appropriate tab of various stains (Figure 1b). The tab was then compared in the patient's mouth for matching. Once accurate matching was obtained, the stain was incorporated using brush on technique [8]. Staining was also incorporated in the lower denture that varied from light to dark stains (Figure 1c). Both dentures were delivered to the patient after giving instructions. The patient was highly satisfied with the esthetic outcome of the denture (Figure 1d).

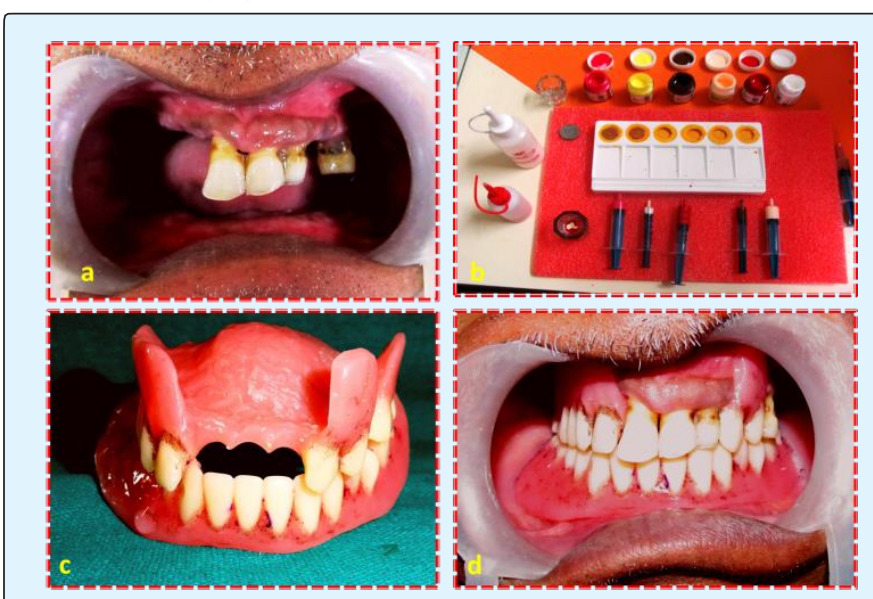

Figure 1: a. Intra oral view of natural teeth showing cervical stains; b. Fabrication of tabs with various shades; c. Stained prosthesis; d. Intra oral view of completed prosthesis with characterization.

\section{Discussion}

Characterizing a prosthesis in terms of staining is a trial and error procedure and there are no standards to it except the technique. Every individual that wants to characterize prosthesis has to make many attempts to achieve the desired results. The coloring agents that are available include ester soluble dyes, water soluble dyes, inorganic and organic pigments and internally colored polymers (Replident, Natur-tint, standard Crayon Kit) $[4,9,10]$ processed denture base acrylic resin can be imparted color by various methods. Coating the resin surface with an adhesive material that contains the desired color is a very simple method, but has its own limitations which are related to the use of adhesive that deteriorates over a period of time especially when it is placed under water. Immersing the processed resin in dye solution allows the dye to be deposited on the surface, but denture base resin is a material that is substantially impervious to ordinary dye solutions and is not attacked by any dye. However, some food products that a patient eats have been able to impart color of denture base resin, but this type of coloring is not permanent and can be removed partly or wholly within a period of days. Imparting a uniform color to the entire denture base is achieved by addition of coloring agent within the monomer and has been used extensively in the fabrication of splints and stents [11-13].

Surface characterization of the denture involves a staining technique that applies pigmentation on the surface layer of the denture which are then processed as integral part of the complete denture prosthesis. Tints may be applied with ultra small camel's hairbrush that is wetted with monomer or by dusting and wetting with the dropper on the external denture base. Different staining kits produce different shades although they are chemically same. The same kit will have a different staining effect on the same quantum of heat cure denture acrylic resin which actually demands detailed studies. The staining kit that was used for this patient required that the area should never be cleaned with dissolving agents that included alcohol. Another significant precaution with surface tints is the lack of abrasion resistance of the stains which makes it compulsory that after packing minimum alteration is done on surface of the denture through finishing and polishing. To minimize that a layer of light cure clear acrylic resin coating may be used $[14,1]$.

\section{Conclusion}

The art of characterizing denture prosthesis should be practiced regularly in clinical practice to meet the realistic expectations of difficult patients. The procedure is simple, but to achieve good results, one needs to practice and develop his own personal spectrum.

\section{Acknowledgements}

The authors would like to acknowledge the efforts of the patient who had to pay extra visits during tab trial procedures.

\section{References}




\section{Open Access Journal of Dental Sciences}

1. Lagdive S, Darekar A, Lagdive S (2012) Review: Characterization of denture bases-Redefining complete denture esthetics. Int J Health Care Biomed Res 1(1): 16-20.

2. Kumar CM, Kumar DR, Sorabh RJ, Krishnan VB (2014) Virtualising natural effects in complete dentures. Sch J App Med Sci 2: 364-368.

3. (2005) The glossary of prosthodontic terms. J Prosthet Dent 94(1): 10-92.

4. Choudhary SC, Craig JF, Suls FG (1975) Characterization of the denture base for non caucasian patients. J Prosthet Dent 33(1): 73-79.

5. Pound E (1951) Esthetic dentures and their phonetic values. J Prosthet Dent 1(1-2): 98-111.

6. Morrow RM, Rudd KD, Rhoads JE (1986) Dental laboratory procedures (complete dentures), $2^{\text {nd }}$ (Edn.), CV Mosby, St. Louis, pp: 545-550.

7. Mattoo K, Shujaurrahman, Arora P (2014) Duplicating palatine rugae in complete denture prosthesis to enhance the relationship between food and taste receptors. Med Res Chron 1(2): 150-155.
8. Powers JL (1953) Brush-on technique in natural coloring of cured cross-linked plastic artificial denture materials. J Prosthet Dent 3(3): 350-353.

9. Winkler S, Vernon HM (1978) Coloring acrylic denture base resins. J Prosthet Dent 40(1): 4-7.

10. Quinlivan JT (1975) Characterization of denture bases. Dent Clin North Am 19: 321- 332.

11. Fonseca EP (1966) The importance of form, characterization, and retention in facial prosthesis. J Pros Den 16(2): 338-343.

12. Griem ML, Robinson JK, Barnhart GW (1964) The use of a soft denture-base material in the management of the post-radiation denture problem. Radiology 82: 320-321.

13. Hawkinson RT (1965) Development of skin surface texture in maxillofacial prosthetics. J Pros Den 15(5): 929-937.

14. Wood GN (1955) Investing In the Upper Half of the Flask. J Prosthet Dent 5(2): 205. 